\title{
FDG PET imaging of paragangliomas of the neck: comparison with MIBG SPET
}

\author{
David J. Macfarlane $1^{*}$, Barry L. Shulkin ${ }^{1}$, Kieran Murphy $2^{*}$, Gregory T. Wolf $3^{*}$ \\ 1 Division of Nuclear Medicine, Department of Internal Medicine, University of Michigan Medical Center, Ann Arbor, Michigan, USA \\ 2 Department of Radiology, University of Michigan Medical Center, Ann Arbor, Michigan, USA \\ 3 Department of Otorhinolaryngology, University of Michigan Medical Center, Ann Arbor, Michigan, USA
}

Received 8 May and in revised form 22 May 1995

\begin{abstract}
Two patients with cervical paragangliomas underwent positron emission tomography (PET) with 2[18F]-fluoro-2-deoxy-D-glucose (FDG). There was marked tumor uptake and retention of FDG. Adjacent salivary gland accumulation of FDG was minimal, though quite prominent with meta-iodobenzylguanidine. FDG PET offers another potentially useful approach to functional imaging of these uncommon tumors, independent of the presence of specific amine uptake mechanisms or cell surface receptors required by other scintigraphic techniques.
\end{abstract}

Key words: Positron emission tomography - Paragangliomas - 2- $\left.{ }^{18} \mathrm{~F}\right]$-Fluoro-2-deoxy-D-glucose - Meta-iodobenzylguanidine - Single-photon emission tomography

\section{Eur J Nucl Med (1995) 22:1347-1350}

\section{Introduction}

Cervical paragangliomas are uncommon neoplasms derived from neural crest cells, and are also referred to as chemodectomas, carotid body tumors, glomus vagale tumors, or glomus tympanicum tumors, depending upon their structure of origin. Bilaterality and association with paragangliomas elsewhere are more common in the setting of familial and endocrine neoplasia syndromes [1]. Distant metastases (12\% of carotid body tumors) and catecholamine synthesis (1\% of paragangliomas) are rare $[1,2]$. The usual history of these tumors is of relentless local growth, eventually producing symptoms by mass effect and progressive compromise of local neurovascular structures [3]. Anatomic imaging techniques re-

\footnotetext{
* Current address: Department of Nuclear Medicine, Royal Brisbane Hospital, Queensland, Australia
}

Correspondence to: B.L. Shulkin, Pediatric Nuclear Medicine, University of Michigan Medical Center, MCHC F3313 Box 0229, Ann Arbor, MI 48109-0229, USA ly upon characteristic spatial relations and high vascularity of the tumors, while modern scintigraphy exploits their neuroendocrine heritage with meta-iodobenzylguanidine (MIBG) and somatostatin receptor agonists. We describe the metabolic imaging of these tumors with positron emission tomography (PET) and $2-\left[{ }^{18} \mathrm{~F}\right]$-fluoro2-deoxy-D-glucose (FDG), and explore the potential usefulness of this technique in comparison with MIBG single-photon emission tomography (SPET).

\section{Materials and methods}

PET images (12 min transmission, 15 min emission) of the neck were obtained beginning $\sim 50 \mathrm{~min}$ following intravenous injection of $370 \mathrm{MBq}(10 \mathrm{mCi})$ FDG using a Siemens Exact whole-body PET scanner. The data were reconstructed into cross-sectional images with a Hann filter and a cutoff frequency of 0.3 . Whole-body images from the top of the skull to the proximal lower extremities were acquired by the method of Dahlbom [4]. SPET images of the neck were obtained $48 \mathrm{~h}$ after intravenous injection of $18.5 \mathrm{MBq}$ $(0.5 \mathrm{mCi})$ of iodine-131 MIBG for $30 \mathrm{~min}$ (patient 1$)$ or $24 \mathrm{~h}$ after intravenous injection of $370 \mathrm{MBq}(10 \mathrm{mCi})$ iodine-123 $\mathrm{MIBG}$ for 15 min (patient 2) using a Picker Prism triple-headed gamma camera rotated through 120 stops. Data were reconstructed into crosssectional images by filtered back projection, a Butterworth filter and a cutoff of $0.2-0.5$. Planar spot images were acquired from the top of the skull to the proximal lower extremities for $20 \mathrm{~min}$ each at $48 \mathrm{~h}$ (patient 1, ${ }^{3}{ }^{3} \mathrm{I}-\mathrm{MIBG}$ ) and $10 \mathrm{~min}$ each at $24 \mathrm{~h}$ (patient 2, 123I-MIBG) using a dual-head gamma camera with highenergy and low-energy collimators respectively.

\section{Results}

Patient 1

A 61-year-old woman with a history of mild asthma and gastroesophageal reflux presented with a firm left neck mass of $\sim 2 \mathrm{~cm}$ diameter which had not resolved despite multiple courses of antibiotics. Panendoscopy of the upper aerodigestive tract was unremarkable. Exploration of 
the left neck revealed a number of small lymph nodes located over the internal jugular vein, biopsies of which yielded only normal lymphoid tissue. However, a $3 \mathrm{~cm}$ by $3 \mathrm{~cm}$, fleshy, violaceous mass was visible immediately deep to the nodes, splaying the internal and external carotid arteries at their origin. A provisional diagnosis of a carotid body tumor was made and the incision closed.
Subsequent urinary catecholamine and metabolite levels were normal.

FDG PET, ${ }^{131}$ I-MIBG planar and SPET, MRI, and carotid angiography studies were performed (Fig. 1). PET images showed intense accumulation of FDG within the tumor (Fig. 1A, D, G). The standardized uptake value (SUV) was 7.7[5]. Salivary gland activity was minimal.
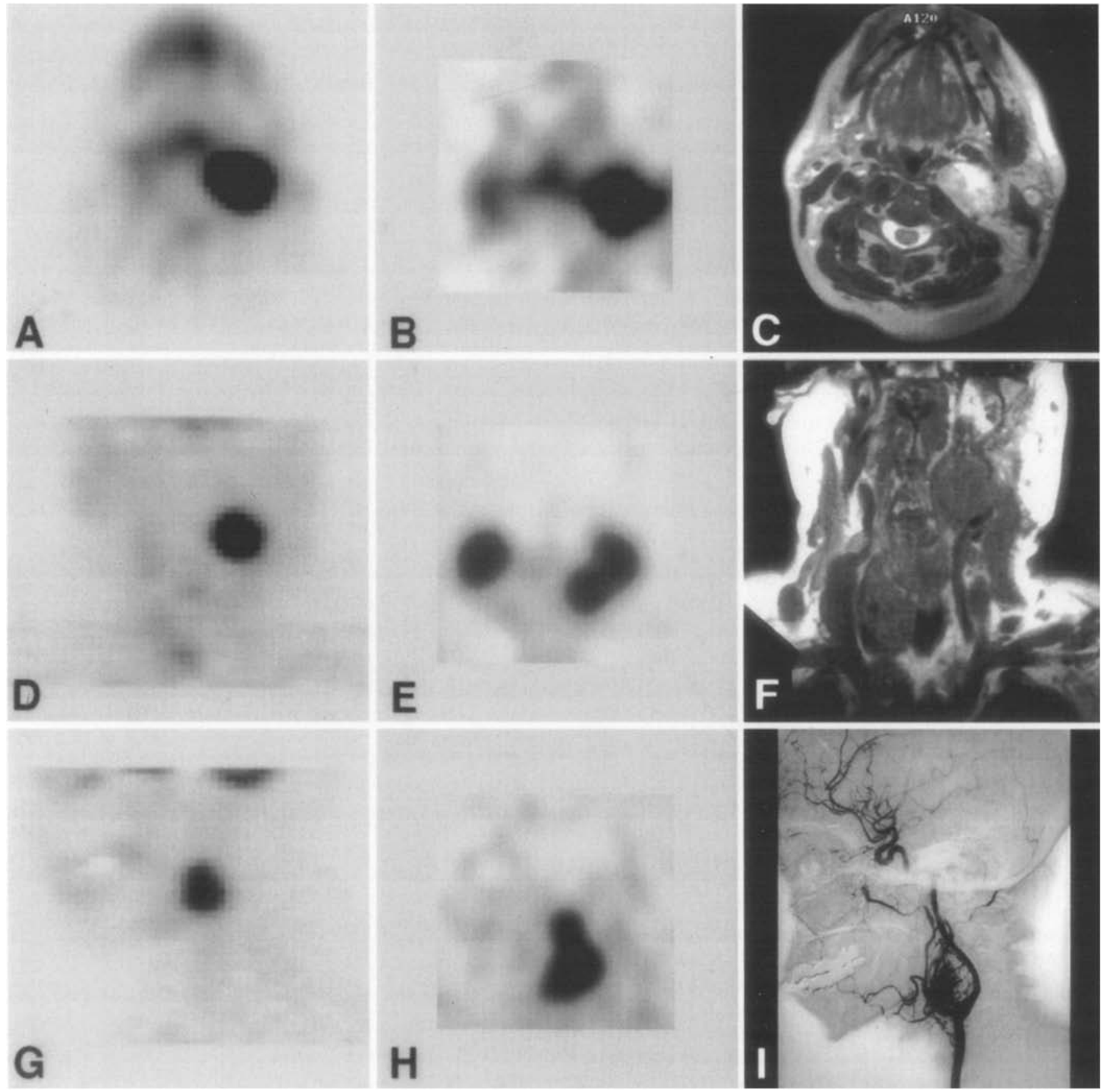

Fig. 1 A-I. Representative images from patient 1. Transverse (top row), coronal (center row), and sagittal sections (bottom row) from FDG-PET (A , D, G), ${ }^{131}$ I-MIBG SPET (B, E, H), MRI (C, F), and carotid angiography studies (I). There is intense uptake of FDG and MIBG within the left neck mass. Normal left parotid salivary gland activity seen on ${ }^{131}$ I-MIBG images is masked by tumor uptake in transverse section $\mathbf{B}$, but evident superior to tumor uptake in coronal section $\mathbf{E}$ and sagittal section $\mathbf{H}$. T1-weighted MR images after ( $\mathbf{C}$, axial) and before ( $\mathbf{F}$, coronal) administration of gadolinium demonstrate the left-sided soft tissue mass with inhomogeneous enhancement following gadolinium injection. Angiography (I) shows a mass with a vascular blush splaying the carotid bifurcation, characteristic of a carotid body tumor 
MIBG SPET images demonstrated intense tracer uptake in the tumor adjacent to the normal left parotid activity (Fig. 1B , E , H). For comparison, the MRI and angiographic studies are also shown in Fig. 1. There was a single lesion within the carotid bifurcation isointense to adjacent soft tissue on precontrast T1-weighted images, with intense heterogeneous "salt and pepper" appearance following gadolinium contrast enhancement (Fig. 1C, F, I)[6]. Whole-body images from both the FDG and MIBG studies were otherwise unremarkable, and showed no evidence of tumors elsewhere.

The resection was uneventful and histologic examination was consistent with a carotid body tumor.

\section{Patient 2}

A 41-year-old otherwise healthy woman presented to her local otolaryngologist describing persistent rhinorrhea, nasal congestion and more recent development of painless right neck swelling. Findings on physical examination were mild right anterior cervical adenopathy, a $3 \times 4$ $\mathrm{cm}$ mass posterior to the angle of the right mandible, sluggish movements of the right vocal cord and marked medial displacement of the right palate. Profuse hemorrhage complicated biopsy of the right neck prominence, which was reported as consistent with a "glomus tumor".

The patient was referred to this institution for further investigation. Carotid angiography revealed a vascular

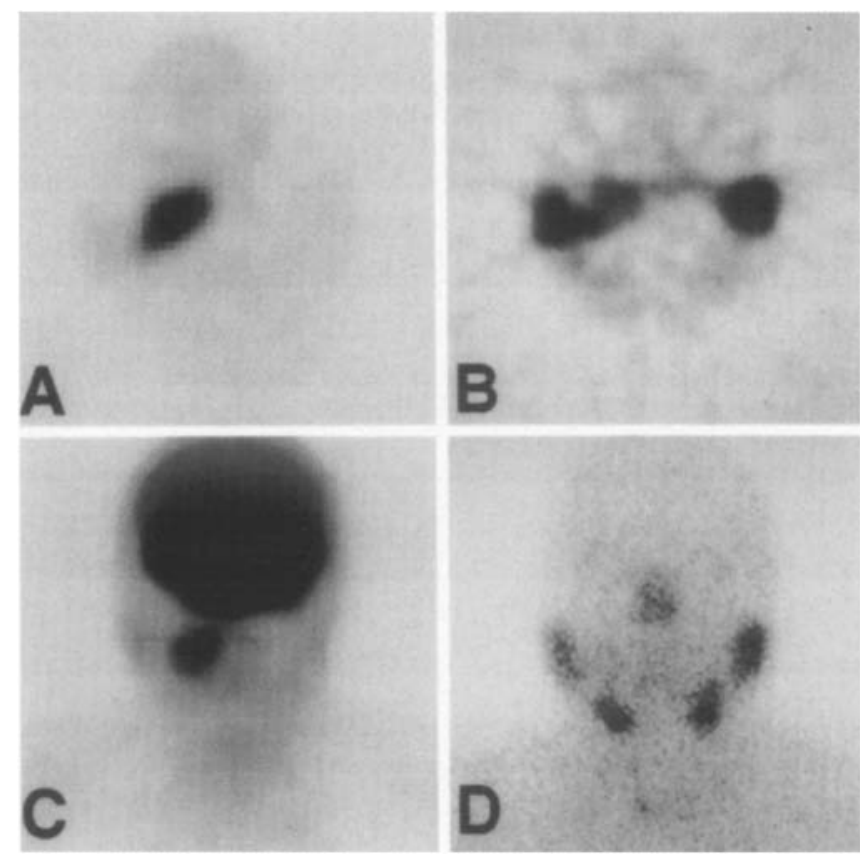

Fig. 2 A-D. Images from patient 2. Transverse (A) and anterior projection (C) images from FDG PET demonstrate an abnormal focus of FDG accumulation in the right neck. The corresponding ${ }^{123}$ I-MIBG transverse section is presented in $\mathbf{B}$. Note that the tumor, readily visible on SPET, is not apparent on a standard anterior planar view (D) lesion at the carotid bifurcation splaying the proximal internal and external carotid arteries, with a characteristic intense vascular blush. An oxygen-15 water quantitative cerebral perfusion study revealed no adverse reduction in perfusion, electroencephalographic changes, or neurologic dysfunction upon occlusion of the right internal carotid artery. FDG PET images showed intense uptake of tracer by the lesion (Fig. 2A , C). The SUV was 5.6. MIBG SPET images showed distinct accumulation within the tumor, medial to the right parotid activity (Fig. 2 B), while planar view showed only subtle asymmetry adjacent to the parotid glands (Fig. 2 D). There was no abnormal uptake elsewhere in the body on either the FDG or MIBG studies to suggest additional neuroendocrine tumors. Plasma and urine catecholamines and metabolites were normal.

The feeding vessels were embolized and the tumor subsequently resected. This required a mandibulotomy, together with partial resection of the right external carotid artery, internal jugular vein, the vagus, hypoglossal, spinal accessory and lingual nerves, and the right cervical sympathetic chain. Histology showed a glomus tumor. After additional revision procedures and renervation of the right vocal cord the patient has satisfactory laryngeal function and cosmetic result.

\section{Discussion}

The typical location and hypervascularity of carotid body tumors has been described by dynamic computed tomography and contrast angiography studies [7]. Magnetic resonance imaging shows focal low signal regions within the tumor on T1-weighted pre-contrast images, a heterogeneous "salt and pepper" appearance on T2weighted images, and intense enhancement following administration of gadolinium [6]

Scintigraphy of these tumors initially comprised simple radionuclide angiography, used in one series to screen kindred members in familial paraganglionoma [ 1 , $8]$. The neural crest origin of the tumors, and the presence of biogenic amine uptake-1 and intracellular storage, was later exploited using MIBG. The low incidence of carotid body tumors has been a factor in the limited reported experience with this agent in carotid body tumors. Van Gils et al. demonstrated tumor uptake of 123IMIBG in 8 of 15 patients with known chemodectomas [2]. The sensitivity with ${ }^{131}$ I-MIBG appears slightly lower, with uptake described in known lesions in four of ten patients [9]. The normal marked salivary gland uptake of MIBG may mask the presence of an adjacent tumor and, thus, SPET should be performed whenever possible [10].

Neuroendocrine tissues frequently express somatostatin receptors. As part of a study of paragangliomas with indium-111 pentetreotide, Kwekkeboom et al. reported successful detection of tumor in 13 of 14 patients with known carotid body tumors [11]. Among 25 patients with paragangliomas, unsuspected additional tumor sites 
were identified in nine. The ability to study the whole body in a single examination is an advantage in a condition where multiple lesions may be present. Our patients were studied prior to commercial availability of this agent.

The substantial elevation of glucose uptake and retention by tumors relative to most non-neoplastic tissue is fundamental to FDG PET imaging in oncology [12]. This approach has been successful in visualizing neuroendocrine tumors even when the specific cellular mechanisms necessary for imaging with MIBG are absent [13]. PET with FDG clearly demonstrated the known tumors in these two patients. These tumors, though benign, were quite metabolically active. Salivary gland uptake of FDG was very low compared to that of MIBG, an advantage given the characteristic location of these tumors. This preliminary report suggests that FDG PET may be a useful adjunct in the detection and staging of cervical paragangliomas.

Acknowledgements. This study was supported by grants 1 R29 CA54216 from the National Cancer Institute and MO1 RR 00042 from the University of Michigan General Clinical Research Center. The authors thank Karen Grahl for typing the manuscript; Robert Ackermann, C.N.M.T., Lisa Cotton, C.N.M.T., Todd Hauser, C.N.M.T., Paul Kison, C.N.M.T., Edward McKenna, C.N.M.T., Steven Pitt, C.N.M.T., Jill Rothley, C.N.M.T., and Andrew Weeden, C.N.M.T., for technological expertise; Shirley Zempel, R.N.C., for assistance with data collection; Brahm Shapiro, M.B., Ch.B., Ph.D., James Sisson, M.D., and Richard Wahl, M.D., for stimulating discussions; and the cyclotron chemistry staff for the production of FDG.

\section{References}

1. Hamilton JR, Barros D'Sa AA. Radionuclide angiography and surgery for familial bilateral chemodectomas. Eur J Vasc Surg 1987; 1: 97-105.

2. van Gils APG, van der Mey AG, Hoogma RP, Falke TH, Moolenaar AJ, Pauwels EK, van Kroonenburgh MJ. Iodine-123metaiodobenzylguanidine scintigraphy in patients with chemodectomas of head and neck region. I Nucl Med 1990; 31: 1147-1155.

3. Wax MK, Briant DR. Carotid body tumors: a review. $J$ Otolaryngol 1992; 21: 277-285.

4. Dahlbom M, Hoffman EJ, Hoh CK, Schiepers C, Rosenqvist G, Hawkins RA, Phelps ME. Whole-body positron emission tomography. Part I. Methods and performance characteristics. J Nucl Med 1992; 33: 1191-1199.

5. Strauss LG, Conti LG. The applications of PET in clinical oncology. J Nucl Med 1991; 32: 623-648.

6. Rippe DJ, Grist TM, Uglietta JP, Fuller GN, Boyko OB. Carotid body tumor: flow sensitive pulse sequences and MR angiography. J Comput Assist Tomogr 1989; 13: 874-877.

7. Brismar J. Angiography of chemodectomas of the neck. Acta Radiol [Diagn] (Stockh) 1980; 21: 689-696.

8. Serafini AN, Weinstein MB. Radionuclide investigation of a carotid body tumor. I Nucl Med 1972; 13: 640-643.

9. von Moll L, McEwan AJ, Shapiro B, Sisson JC, Gross MD, Lloyd R, Beals E, Beierwaltes WH, Thompson NW. Iodine131 MIBG scintigraphy of neuroendocrine tumors other than pheochromocytoma and neuroblastoma. J Nucl Med 1987; 28: 979-988.

10. Weissman AF, Gonzalez CE, Shapiro B, Shulkin B, Francis IR, Leach K. Multiple chemodectomas. Carotid body tumor masked by salivary gland uptake on I-123 MIBG scintigraphy. Clin Nucl Med 1994; 19: 527-531.

11. Kwekkeboom DJ, van Urk H, Pauw BK, Lamberts SW, Kooij PP, Hoogma RP, Krenning EP. Octreotide scintigraphy for the detection of paragangliomas. I Nucl Med 1993; 34: 873-878.

12. Wahl RL, Hutchins GD, Buchsbaum DJ, Liebert M, Grossman HB, Fisher S. Fluorine-18-2-deoxy-2-fluoro-D-glucose (FDG) uptake into human tumor xenografts: feasibility studies for cancer imaging with PET. Cancer 1991; 67: 1544-1549.

13. Shulkin BL, Koeppe RA, Francis IR, Deeb GM, Lloyd RV, Thompson NW. PET FDG localization of pheochromocytomas which fail to accumulate MIBG. Radiology 1993; 186: $711-715$. 